\title{
Prefrontal Cortex Function in the Representation of Temporally Complex Events
}

\author{
Philip G. F. Browning and David Gaffan \\ Department of Experimental Psychology, Oxford University, Oxford OX1 3UD, United Kingdom
}

\begin{abstract}
The frontal cortex and inferior temporal cortex are strongly functionally interconnected. Previous experiments on prefrontal function in monkeys have shown that a disconnection of prefrontal cortex from inferior temporal cortex impairs a variety of complex visual learning tasks but leaves simple concurrent object-reward association learning intact. We investigated the possibility that temporal components of visual learning tasks determine the sensitivity of those tasks to prefrontal-temporal disconnection by adding specific temporal components to the concurrent object-reward association learning task. Monkeys with crossed unilateral lesions of prefrontal cortex and inferior temporal cortex were impaired compared with unoperated controls at associating two-item sequences of visual objects with reward. The impairment was specific to the learning of visual sequences, because disconnection was without effect on object-reward association learning for an equivalent delayed reward. This result was replicated in monkeys with transection of the uncinate fascicle, thus determining the anatomical specificity of the dissociation. Previous behavioral results suggest that monkeys represent the two-item serial compound stimuli in a configural manner, similar to the way monkeys represent simultaneously presented compound stimuli. The representation of simultaneously presented configural stimuli depends on the perirhinal cortex. The present experiments show that the representation of serially presented compound stimuli depends on the interaction of prefrontal cortex and inferior temporal cortex. We suggest that prefrontal-temporal disconnection impairs a wide variety of learning tasks because in those tasks monkeys lay down similar temporally complex representations.
\end{abstract}

Key words: discrimination; inferotemporal cortex; learning; macaque; memory; prefrontal cortex

\section{Introduction}

The frontal cortex and inferior temporal cortex are strongly functionally interconnected (Fuster et al., 1985). In monkeys, one way to investigate the significance of this interaction is to assess cognitive function after a surgical disconnection of prefrontal cortex from inferior temporal cortex. Inferior temporal cortex is essential for the identification of objects (Tanaka, 1996), and so frontal-temporal disconnection achieved by, for example, a unilateral lesion of prefrontal cortex in one hemisphere and a unilateral lesion of inferior temporal cortex in the opposite hemisphere impairs those visual learning tasks that depend on prefrontal cortex having access to visual information represented in inferior temporal cortex. Monkeys with frontal-temporal disconnection made in this way, by crossed unilateral lesions, are impaired on tasks such as visual conditional learning, in which particular initial cues must be associated with subsequent choice stimuli, and visual strategy implementation, in which different classes of stimuli must be associated with different patterns of responding (Bussey et al., 2002b; Gaffan et al., 2002). In addition, a recent experiment has shown that disconnection abolishes learning in a

Received Nov. 16, 2007; accepted March 3, 2008

This work was supported by the Medical Research Council. We thank Mark G. Baxter for running the anesthesia for our surgeries.

Correspondence should be addressed to Philip G. F. Browning, Department of Experimental Psychology, Oxford University, South Parks Road, 0xford OX1 3UD, UK. E-mail: philip.browning@psy.ox.ac.uk.

D01:10.1523/JNEUROSCI.0633-08.2008

Copyright $\odot 2008$ Society for Neuroscience $\quad$ 0270-6474/08/283934-07\$15.00/0 discrimination learning set paradigm, a paradigm that encourages the formation of prospective memories (Murray and Gaffan, 2006; Browning et al., 2007). Frontal-temporal disconnection nevertheless does not impair concurrent object-reward association learning, indicating that simple associative learning processes are preserved and suggesting that there is a qualitative difference between frontal cortical involvement in concurrent object-reward association learning and its involvement in other tasks (Parker and Gaffan, 1998a; Gaffan et al., 2002).

We examined this difference further by investigating the possibility that the temporal components of the tasks impaired by prefrontal cortical lesions are critical for their successful performance. In two experiments, we compared the ability of unoperated control monkeys and monkeys with prefrontal-temporal disconnection to learn concurrent object-reward associations with additional temporal components in two tasks shown in Figure 1. In one task (task 2I), the visual stimuli presented for learning were two-item sequences of visual objects. In another task (task UD), an unfilled delay was imposed between the chosen object and the rewards for which the monkeys worked. Task UD was identical to task 2I except that the second object in the sequence was replaced by an unfilled delay. This allowed a valid comparison between task 2I and task UD concerning the significance of the sequential presentation of items in task 2I. The first experiment compared learning of problems in these tasks in control monkeys and in monkeys who had received crossed unilateral ablations of prefrontal and inferior temporal cortex. 
Task 2I

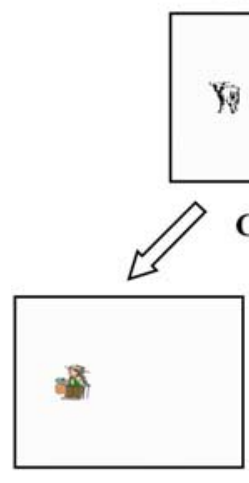

Reward

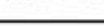

9

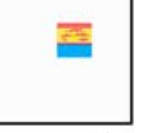

Choice

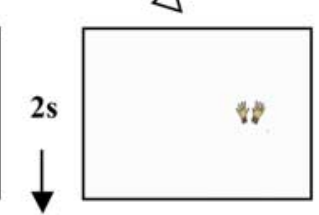

No reward
Task UD

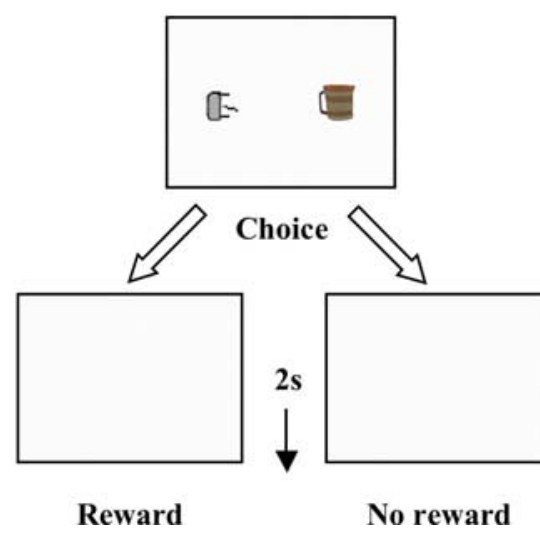

Figure 1. Behavioral tasks. Each panel shows a single discrimination problem from each behavioral task. In each testing session, monkeys learned by trial and error which stimuli led to reward. In each experiment, monkeys learned sets of problems from the two tasks in a counterbalanced manner. In task 21 (left), monkeys associated two-item sequences of objects with reward. In task UD (right), monkeys associated single items with reward separated by an unfilled delay of $2 \mathrm{~s}$.

The second experiment assessed learning in the same tasks in monkeys with transection of the uncinate fascicle. Transecting this pathway reliably disconnects frontal cortex from temporal cortex (Ungerleider et al., 1989) and impairs conditional learning with visual cues but leaves concurrent object-reward association learning intact. This suggests that the qualitative difference between the involvement of frontal-temporal interaction in simple associative learning and in other, more complex tasks may depend more specifically on the uncinate fascicle (Eacott and Gaffan, 1992; Gutnikov et al., 1997). Thus, assessing associative learning with additional temporal components after both methods of frontal-temporal disconnection allowed us both to test the anatomical specificity of frontal-temporal interaction and to confirm the reliability of the postoperative effects seen.

\section{Materials and Methods}

Subjects. All experiments were conducted in accordance with the UK Home Office regulations. Six male cynomolgus monkeys (Macaca fascicularis) of mean age 4 years and 9 months and of mean weight $5.3 \mathrm{~kg}$ at the start of training were used. Monkeys were all housed together in large enclosures with water provided ad libitum. All monkeys had previously taken part in experiments in which they learned concurrent visual discriminations, and the training history of all monkeys was identical. In experiment 1 , three monkeys were unoperated controls (monkeys A-C), and three monkeys had unilateral prefrontal ablation in one hemisphere combined with a unilateral inferior temporal cortex ablation in the other hemisphere, thus achieving the frontal-temporal disconnection (monkeys D-F). The hemisphere (left or right) that received each ablation was counterbalanced across monkeys. Both groups entered a separate experiment in which they learned concurrent object discriminations and reversals (our unpublished data). The operated monkeys were then perfused. In experiment 2, the three monkeys who had previously been controls were tested before and after surgery to transect the uncinate fascicle.

Apparatus. The apparatus consisted of a computer-controlled touchsensitive monitor ( $380 \mathrm{~mm}$ wide by $280 \mathrm{~mm}$ high) on which the stimulus material was presented. The monkey sat in a wheeled transport cage 150 $\mathrm{mm}$ from the screen and made choices between stimuli by reaching out through the bars of the cage. Touches were registered by the computer, and banana-flavored pellets (190 mg; supplied by Noyes, Lancaster, NH) were delivered as rewards for correct responses to a food hopper placed centrally below the monitor. A single large food reward was delivered at the end of each session by the opening of a box set to one side of the centrally placed food hopper. The opening of the box with the large food reward, like all other aspects of the events and the experimental contingencies during any session of training, was under computer control.

Stimulus material. The stimuli used were clipart "objects" 128 by 128 pixels in size presented on a white background on the touchscreen that had a resolution of 800 pixels wide by 600 pixels high. The monkey chose between presented objects by touching one of them. For each set of problems, novel objects were chosen randomly without replacement by the computer from a pool of 6100 unique objects. For this choice, a unique random seed was used for each individual monkey so that the variance among problem sets produced by differences between the objects used in particular problem sets was confounded with the variance between individual monkeys. Figure 1 shows examples of these clipart objects.

Task procedure. The behavioral tasks used in each experiment (described in Fig. 1) were based on the concurrent object-reward association learning task. On any trial, two images were displayed on the left and right sides of the touchscreen. Any such pair of images constituted a discrimination problem in which one image was designated as correct and the other incorrect. The left-right position of the images was random. The monkeys chose between the images by touching within their boundaries and learned by trial and error which stimuli led to reward. In task 2I, monkeys associated two-item sequences of objects with reward. In this task, after a choice had been made the delivery of reward or nonreward occurred after the presentation of another object in the same left-right position as the image chosen. This object remained on the screen for $2000 \mathrm{~ms}$. There was no requirement to touch the second object in the sequence, although all monkeys tended to rapidly touch it, especially in the initial stages of learning a new set. [Over the course of experiment 1, monkeys made a mean of 1.75 responses per trial to this object in the first session with a new set and remained in contact with the object for a mean period of 795 $\mathrm{ms}$, indicating that they were generally attentive to the two-item sequence (no difference between groups: both $t_{(4)}<1$ ).] Previous behavioral studies have shown that monkeys readily learn about serial compound stimuli, even when the individual items are acquired incidentally (Gaffan and Dickinson, 2008). The second object in the sequence was unique to each choice stimulus such that a choice of the $S-$ would begin the $\mathrm{S}-$ sequence, whereas a choice of the $\mathrm{S}+$ would begin the $\mathrm{S}+$ sequence. In task UD, monkeys associated single items with reward, where the choice and delivery of reward or nonreward were separated by an unfilled delay of $2 \mathrm{~s}$. Correct choices were rewarded with the delivery of $190 \mathrm{mg}$ reward pellets.

Trials were separated by an intertrial interval of $10 \mathrm{~s}$ after a correct choice and of $20 \mathrm{~s}$ after an incorrect choice. Discriminations were learned concurrently in sets of four problems. [Although this small number could potentially have resulted in the formation of a discrimination learning set, thus complicating our interpretation (Browning et al., 2007), there was no increase in the within-problem rate of learning over the course of the experiment evidenced by an absence of any linear effect of set number on performance in either task by unoperated monkeys (all $F_{(1,2)}<1$ ). This can be seen in supplemental Table 1 (available at www. jneurosci.org as supplemental material).] Each presentation of the four problems together constituted a block of trials in which each problem was presented once. The order of problems within each block was randomized. Daily sessions consisted of 30 blocks of trials unless the session was the first session with that set, in which case sessions consisted of 25 blocks. [All monkeys had previously formed part of an experiment in which they learned four-item sequences (our unpublished data). These four-item sequences were difficult for the monkeys to learn, and performance was frequently poor in the first session. In that experiment, therefore, the first session with a set, in which many errors were made, was shortened to prevent the monkeys getting overly frustrated. When the 
monkeys entered the present experiment, with two-item sequences, we wanted to ensure the task procedure was identical in every way bar the number of items presented in a sequence. This ensured that our data were comparable with our previous investigations.] The monkeys continued daily sessions with a set of four problems until a criterion was met. This criterion was $90 \%$ correct choices in the whole session, in sessions after the first session with a new set, or of $90 \%$ correct choices after the first block of the session if that session was the first with that set. Once the criterion was met, learning began on the next set of novel problems. After a correct choice on the last trial in a session, a lunchbox containing the full daily diet of a monkey opened. An incorrect choice on this trial meant that another problem from the set was presented until a correct choice was made and the lunchbox obtained. All these contingencies, including the opening of the lunch box, were controlled by computer.

Monkeys learned a total of eight sets of problems in task 2I and eight sets of problems in task UD. These sets were presented in a counterbalanced manner as follows. Monkeys first learned four sets of task 2I, then eight sets of task UD, and finally the remaining four sets of task 2I. This design was adopted to minimize general practice effects or other more specific learning effects particular to any one task, such as the development of a learning set (see above). After completing experiment 1, the unoperated control monkeys later performed experiment 2. They received bilateral section of the uncinate fascicle, and their postoperative rate of learning over eight new sets of problems in tasks 2I and UD, presented in the same counterbalanced manner as in experiment 1 , was compared with their preoperative learning rate in those tasks. The dependent measure of errors to criterion was transformed to a natural logarithmic scale before appropriate statistical tests were performed.

Surgical procedures. Operations were performed in a single stage under sterile conditions. The monkeys were anesthetized throughout surgery with barbiturate (thiopentone sodium) administered through an intravenous cannula. Monkeys were maintained in a state of deep anesthesia by monitoring pulse rate, blood oxygenation, body temperature, and peripheral reflexes. The ablations were made by aspiration with the aid of an operating microscope. Monkeys received buprenorphine hydrochloride $(0.01 \mathrm{mg} / \mathrm{kg})$ as an analgesic and amoxicillin $(8.75 \mathrm{mg} / \mathrm{kg})$ to prevent infection, daily, for 1 week postoperatively.

Unilateral prefrontal ablation. In the hemispheres with prefrontal ablation, the intention was to remove prefrontal cortex while sparing cingulate, supplementary, and premotor cortices. The posterior limit of the lesion on the lateral surface of the frontal lobe followed an approximate line joining the tips of the ascending and descending limbs of the arcuate sulcus and the posterior end of the principal sulcus. The entire arcuate sulcus was to be spared. From the anteriormost point of the ascending limb of the arcuate sulcus, the boundary extended medially and then ventrally into the interhemispheric fissure. Medially, this boundary remained at this same anterior-posterior level up to the crown of the cingulate sulcus. The entire cingulate cortex was to be spared. All cortex forward of this boundary was removed, including the cortex in both banks of the principal sulcus. From the anteriormost point of the descending limb of the arcuate sulcus, the boundary extended downward to the lateral sulcus. The entire orbital surface of the frontal lobes was removed, including the cortex in the medial and lateral orbital sulci. This orbital removal was extended onto the ventral part of the medial surface of the hemisphere as far dorsally as the rostral sulcus. The white matter surrounding the corpus striatum and the striatum itself was to be left intact.

Unilateral inferior temporal cortex ablation. The boundaries of the unilateral inferotemporal ablation were identical to those in previous experiments with this lesion in our laboratory (Browning et al., 2007). The arch of the zygoma was removed, and the temporal muscle was detached from the cranium and retracted. A bone flap was raised and extended with a rongeur, and then the dura mater was cut. The extent of the intended lesion is shown in Figure 1. The ablation extended from the fundus of the superior temporal sulcus to the fundus of the rhinal sulcus, and posteriorly included both banks of the anterior part of the occipitotemporal sulcus. The posterior limit of the ablation was a line drawn perpendicular to the superior temporal sulcus, $5 \mathrm{~mm}$ anterior to the inferior occipital sulcus. The anterior limit of the ablation was the ante- rior tip of the superior temporal sulcus and a line drawn round the pole from that tip to the rhinal sulcus. Within these limits, all the cortex was removed, including both banks of the anterior and posterior middle temporal sulci.

Uncinate fascicle transection. Uncinate fascicle transection was achieved using the same procedure as Ungerleider et al. (1989). The zygomatic arch was removed to allow the temporal muscle to be retracted to expose the bone overlying the lateral sulcus. The bone overlying and surrounding the lateral sulcus was removed with a rongeur. The dura was cut, and the pia mater along the inferior lip of the lateral sulcus was cauterized from the temporal pole to the level of the central sulcus, leaving intact crossing arteries where possible. The cortex within the lower bank of the lateral sulcus was removed, exposing the pia mater in the lower limb of the insula. The underlying white matter of the uncinate fascicle adjacent to the foot of the insula was sectioned by cautery and aspiration. The removal was performed bilaterally, and when the surgery was complete, overlying tissue was closed in layers. Monkeys were allowed 12-14 d for postoperative recovery.

Histology. At the completion of all behavioral testing, subjects were deeply anesthetized and transcardially perfused with physiological saline followed by $10 \%$ formalin. The brains were extracted and cut on a freezing microtome in $50 \mu \mathrm{m}$ sections in the coronal plane. Figure 2 shows four actual sections and their corresponding reconstructions from monkey D. Reconstructions from monkeys $\mathrm{E}$ and $\mathrm{F}$ at the same anteriorposterior level (Paxinos et al., 2000) are also shown. The estimated extent of the lesion in each monkey was plotted onto scanned sections taken from an unoperated macaque and highlighted in red. These ablations were essentially as intended except for partial sparing of perirhinal cortex in monkey $\mathrm{D}$ and unintended damage limited to the anterior cingulate gyrus in monkeys $\mathrm{E}$ and $\mathrm{F}$. The ablations in experiment 2 were intended to section the uncinate fascicle. The histological results from these monkeys are shown in Figure 4. Sections were taken at the level of the anterior commissure, and the expected degeneration of this structure can be seen in the section taken from monkey A. In the adjacent reconstruction, the aspirated tissue is outlined on an intact macaque section in red. Reconstructions from other monkeys are also shown. As stated above, exposure of the uncinate fascicle necessarily requires the removal of some cortex from the inferior bank of the lateral sulcus and from the lower foot of the insula. In addition to this expected cortical damage, monkey A and monkey B sustained minor unintended damage unilaterally. Monkey A had minor damage to the superior temporal gyrus in the left hemisphere, and monkey $\mathrm{B}$ had damage to the fundus of the superior temporal sulcus in the left hemisphere. Monkey $\mathrm{C}$ had no unintended cortical damage. All the uncinate fascicle transections were complete.

\section{Results}

The behavioral results from experiment 1 are shown in Figure 3. This figure shows the mean number of errors to criterion made by control monkeys and monkeys with crossed unilateral lesions of prefrontal and inferior temporal cortex in the two tasks over eight sets of problems. Overall monkeys with frontal-temporal disconnection made $\sim 2.9$ times more errors in task 2 I than unoperated control monkeys. Learning in the two-item task was compared with task UD, in which a single object must be associated with delayed reward. Both groups of monkeys made a similar number of errors overall in this task. The data shown in Figure 3 were analyzed by ANOVA using "task" as the within-subjects factor and "group" as the between-subjects factor. This comparison revealed a significant task-by-group interaction $\left(F_{(1,2)}=\right.$ $23.87 ; p=0.008)$. Pairwise comparisons indicated that the monkeys with frontal-temporal disconnection learned problems in task 2I significantly slower than controls $\left(t_{(4)}=5.095 ; p=0.007\right)$ but learned problems in task UD at a rate comparable with that of control monkeys $\left(t_{(4)}=1.693 ; p=0.166\right)$. The specificity of the impairment was particularly striking in this experiment, because control animals learned more slowly in the unfilled delay task than in the two-item task $\left(t_{(2)}=5.502 ; p=0.031\right.$, paired $t$ test $)$. 


\section{A) PREFRONTAL ABLATION}

INFERIOR
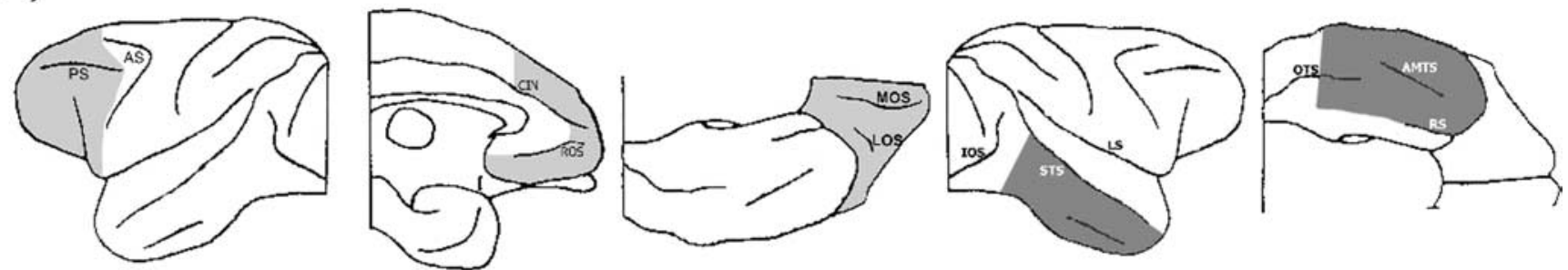

B)

$+35$

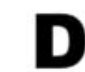

D
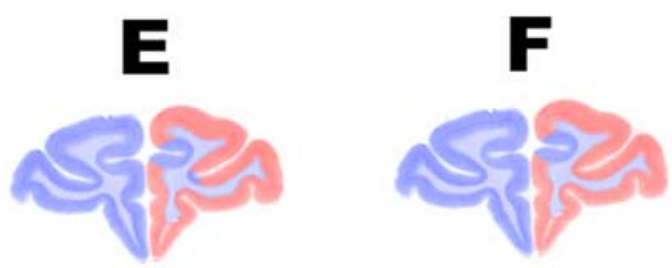

$+30$
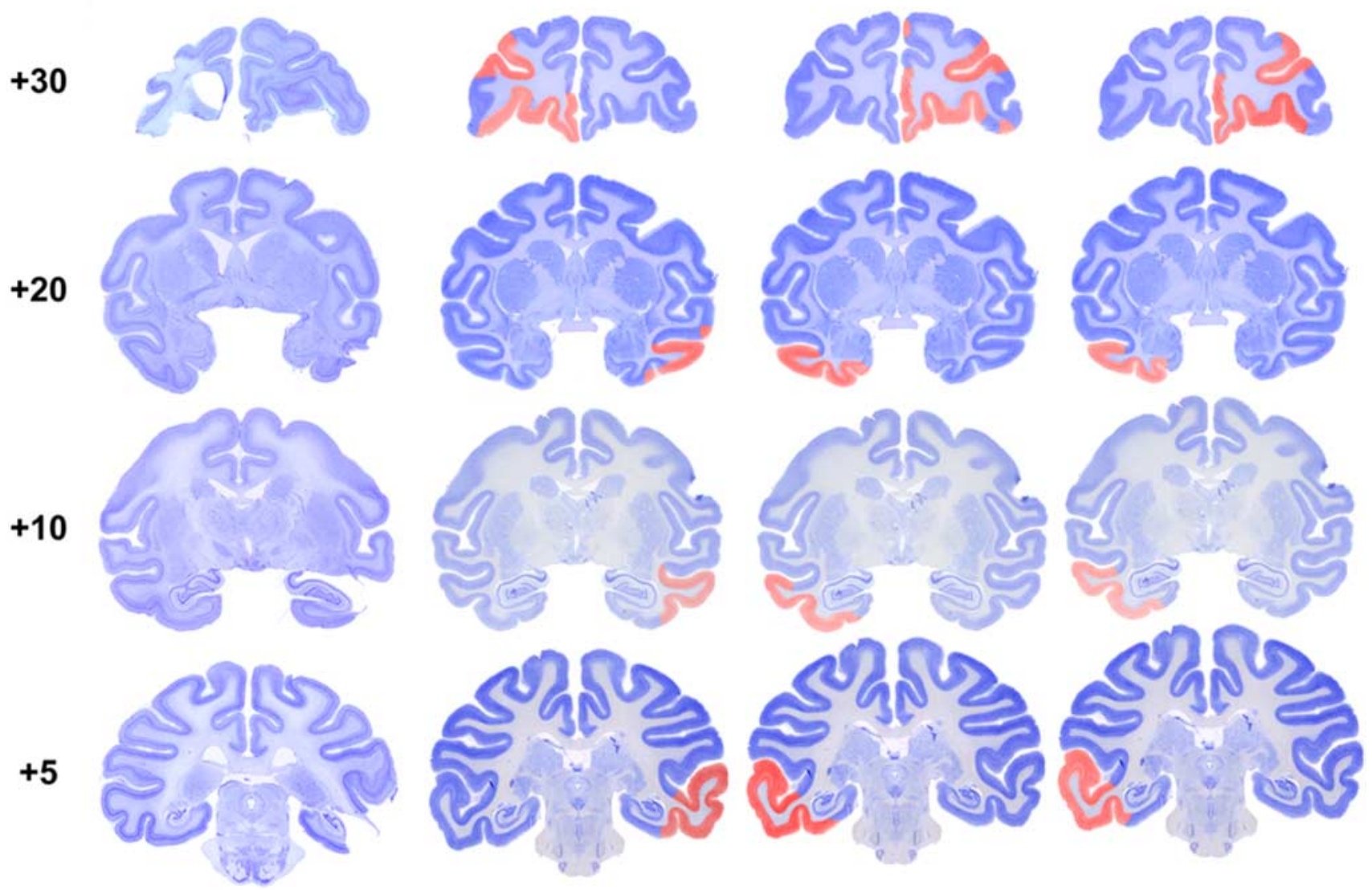

Figure 2. Histological reconstructions. A, Intended area of the asymmetric ablations in monkeys with disconnection of prefrontal cortex from inferior temporal cortex (group PFC $\times$ IT) shown from lateral, ventral, and medial views. The shaded areas indicate the areas of intended removal; the unilateral prefrontal ablation is shown in light gray, and the unilateral inferior temporal ablation is shown in dark gray. Monkeys received a prefrontal ablation in one hemisphere and an inferotemporal ablation in the opposite hemisphere. The hemisphere (left or right) that received each ablation was counterbalanced across monkeys. PS, Principal sulcus; AS, arcuate sulcus; CIN, cingulate sulcus; ROS, rostral sulcus; MOS, medial orbital sulcus; LOS, lateral orbital sulcus; IOS, inferior occipital sulcus; STS, superior temporal sulcus; LS, lateral sulcus; OTS, occipitotemporal sulcus; AMTS, anterior middle temporal sulcus; RS, rhinal sulcus. $B$, Coronal sections of the actual and reconstructed lesions of monkeys from group PFC $\times$ IT at different anterior-posterior levels [estimated from Paxinos et al. (2000)]. The first column shows four actual sections taken from monkey D. The second column shows a reconstruction, colored in red, of the extent of the asymmetric prefrontal and inferotemporal removal in monkey D represented on cresyl violet-stained sections taken from a normal macaque brain. The remaining two columns show similar reconstructions from monkeys $E$ and $F$. Monkey $D$ had left hemisphere prefrontal ablation and right hemisphere inferior temporal ablation. Monkeys $\mathrm{E}$ and $\mathrm{F}$ had right hemisphere prefrontal ablation and left hemisphere inferior temporal ablation.

The impairment in task 2I was stable over the eight sets learned, evidenced by the absence of a linear effect of set number on performance of both operated and unoperated monkeys (see supplemental Table 1, available at www.jneurosci.org as supple- mental material) (unoperated: $F_{(1,2)}<1$; operated: $F_{(1,2)}=1.85$; $p=0.306)$.

In the second experiment, using the monkeys that had formed the normal control group, we assessed learning after disconnec- 


\section{Experiment 1}

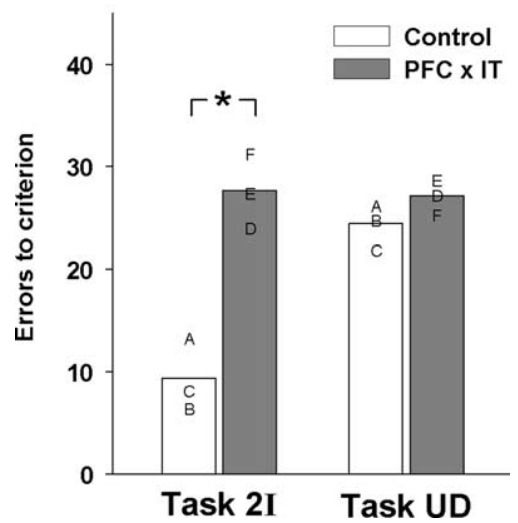

Figure 3. Experiment 1. Learning proficiency in each task is shown by the mean number of errors per set of visual discrimination problems; group means are shown by bars, and individual means are shown by letters. Letters correspond to each monkey's histologically verified lesions shown in Figure 2. Monkeys with disconnection of prefrontal cortex from inferior temporal cortex (shaded bars; $\mathrm{PFC} \times \mathrm{IT}$ ) were impaired relative to controls (unshaded bars) at associating two-item sequences of visual objects with reward (Task 2l). The same monkeys were not impaired at associating single items with reward after an unfilled delay (Task UD). ${ }^{*} p<0.05$.

tion accomplished by surgically sectioning the white matter of the uncinate fascicle. This disconnection produced a pattern of impairment very similar to that seen in the first experiment with crossed unilateral ablations using the same tasks. This is shown in Figure 4. In task 2I, monkeys made 2.2 times more errors postoperatively, but in task UD, the number of errors was comparable with that made preoperatively. This pre-post difference in mean errors per set was significantly greater in task 2I than in task UD, as predicted $\left(t_{(2)}=3.586 ; p=0.035\right.$, one-tailed). Similar to experiment 1 , the impaired postoperative performance on task $2 \mathrm{I}$ was stable over the eight sets tested; no linear effect of set number on performance was evident $\left(F_{(1,2)}<1\right)$.

\section{Discussion}

Previous experiments have shown that a surgical disconnection of prefrontal cortex from inferior temporal cortex achieved either by crossed unilateral ablations of prefrontal and inferior temporal cortex or by transection of the uncinate fascicle does not impair simple object-reward associative learning (Eacott and Gaffan, 1992; Parker and Gaffan, 1998a; Gaffan et al., 2002). In the present experiments, we added temporal components to the standard object-reward associative learning task and assessed learning rates in monkeys with prefrontal-temporal disconnection. The first experiment compared control monkeys with monkeys that had received prefrontal-temporal disconnection accomplished by a unilateral ablation of prefrontal cortex in one hemisphere and a unilateral ablation of inferior temporal cortex in the opposite hemisphere. The second experiment compared preoperative and postoperative performance of monkeys with a surgical transection of the uncinate fascicle. Previous studies have shown that both disconnections, while leaving simple objectreward association learning intact, impair more complex visual memory tasks, including object-in-place scene learning and conditional learning (Eacott and Gaffan, 1992; Parker and Gaffan, 1998a,b; Bussey et al., 2002b; Browning et al., 2005; Browning and Gaffan, 2008). However, in the present experiment, monkeys with either form of disconnection were substantially impaired simply when the stimuli presented for association learning were extended in time to form two-item serial visual compounds. In contrast, association learning in the unfilled delay task, in which only a single object must be associated with reward in any one trial, proceeded at a normal rate. The normal learning rates in disconnected monkeys on the unfilled delay task show that a disruption of simple short-term memory processes is not the cause of the impairments seen in the two-item task. Thus, overall, prefrontal-temporal disconnected monkeys were consistently and substantially impaired in associating serial visual compounds with reward, and the impairment was clearly specific to the learning of serial visual compound discriminations, because these disconnections were without effect on single-item associative learning for an equivalent delayed reward.

Neither of our experiments included an operated control group, and this leaves each of the results open to a possible alternative explanation. In experiment 1, with crossed unilateral lesions, it is possible that the effects seen were effects of unilateral lesions alone rather than effects specifically of crossed unilateral lesions. Previous experiments that have used the technique of prefrontal-temporal disconnection by crossed unilateral lesions have sometimes included a test of unilateral temporal or unilateral prefrontal lesions alone, and have always found that the unilateral lesions alone do not produce the impairments seen after disconnection (Gaffan and Murray, 1990; Gaffan and Hornak, 1997; Parker and Gaffan, 1998a,b; Easton and Gaffan, 2001; Bussey et al., 2002b; Gaffan et al., 2002; Browning et al., 2005, 2007). However, because these experiments used visual learning and memory tasks that were not identical to the present task, we cannot rule out the possibility that unilateral lesions alone may impair serial visual compound learning. Similarly, in experiment 2 , with uncinate fascicle section, it is possible that the effects seen were effects of the partial removal of cortex in the superior temporal gyrus, which is necessary to expose the uncinate fascicle for surgical section. Again, some previous experiments on uncinate fascicle section did include a control group with superior temporal gyrus removals and uncinate fascicle intact, and saw no impairment in visual learning and memory tasks in these operated control monkeys (Eacott and Gaffan, 1992), but, because the behavioral tasks in those experiments did not include the present behavioral task, we again cannot rule out the possibility that this control operation may impair serial visual compound learning. However, if in fact the effects we saw were not caused by prefrontal-temporal disconnection, then serial visual compound learning, unlike other visual learning and memory tasks, must be sensitive both to unilateral prefrontal or temporal lesions alone and to bilateral partial removals of the superior temporal gyrus. This joint alternative explanation of our results seems to us improbable, but it is important to note that we cannot rule it out.

The present experiment did not measure the monkeys' learning about the second items in two-item sequences. Previous experiments, however, have done so, and have demonstrated conclusively that monkeys acquire two kinds of knowledge about the items after the first item in sequences of this kind, presented exactly as in the present experiment. First, Gaffan and Dickinson (2008) showed that, after monkeys learned two-item sequences, subsequent changes in the value of one item taken from such a sequence generalized to the other item taken from the sequence, a generalization that must be mediated by a learned association between the two items, acquired during the initial learning of the two-item sequence. We suggest that this learned association of the second item with the first item, demonstrated in Gaffan and Dickinson's study (2008), allows the monkey to bridge the delay between the initial choice and the reward outcome in the twoitem sequence task. Second, an unpublished study by S. A. Gut- 

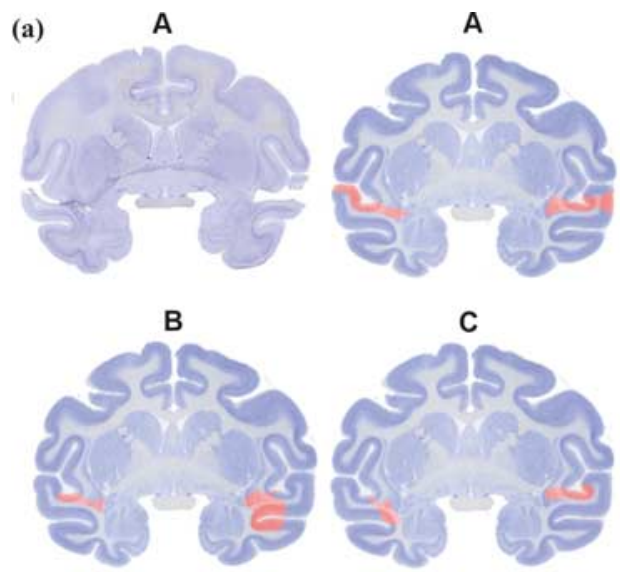

(b)

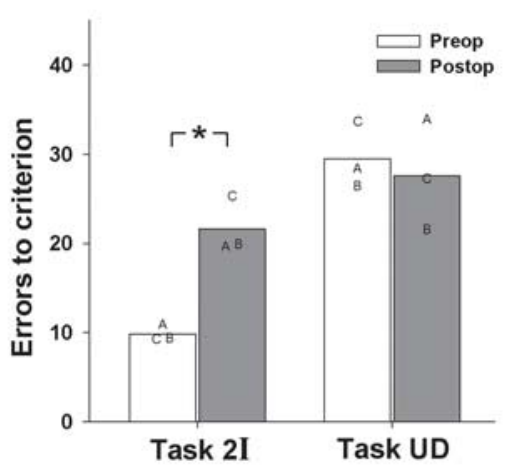

Figure 4. Experiment 2.a, Coronal sections of the actual and reconstructed lesions in monkeys A-C, who had disconnection of prefrontal and inferotemporal cortex by surgical section of the uncinate fascicle. $\boldsymbol{b}$, Learning proficiency on each task is shown by the mean number of errors per set of visual discrimination problems; group means are shown by bars, and individual means are shown by letters. Letters correspond to each monkey's histologically verified lesions shown in $\boldsymbol{a}$. Similar to the monkeys in experiment 1 , these monkeys were impaired at associating two-item sequences of visual objects with reward ( $\boldsymbol{b}$, task $2 \mathrm{l})$ but were not impaired at associating single items with reward after an unfilled delay ( $\boldsymbol{b}$, task UD), compared with their preoperative performance (unshaded bars). ${ }^{*} p<0.05$.

nikov and D. Gaffan gave monkeys transfer tests with the separate elements of three-item serial compounds after the compounds were learned. For example, after learning the sequence A-B-Creward, the monkey was given single-trial transfer tests of preference between A alone and a trial-unique novel object, between $\mathrm{B}$ alone and a trial-unique novel object, and between $\mathrm{C}$ alone and a trial-unique novel object. In 100 such one-trial tests of each type (A, B, and C), given to three monkeys that had learned 100 such three-item discriminations, monkeys chose A on $70.3 \%$ of onetrial transfer tests, B on $84 \%$, and C on $87.4 \%$. (In similar transfer tests with the separate elements of the unrewarded three-item sequences, the monkeys chose the novel object in preference to the element taken from the unrewarded sequence.) These results show that, during the learning of the three-item sequences, the monkeys learned a reward association to the items after the first item, and that this association was stronger than the reward association to the first item itself. In summary, these two kinds of learning about the items after the first item in a multi-item sequence discrimination, namely item-item association and itemreward association, demonstrate that monkeys in learning multiitem sequences attend to and learn about the items after the first item in any sequence.

Monkeys' learning of serial compound visual discriminations could be explained simply by their acquisition of two-term associations. For example, a monkey exposed to the sequence A-Breward could learn the two associations A-B and B-reward. A more plausible explanation, however, is that monkeys learn these discriminations by acquiring a configural representation of the whole event A-B-reward. That is because the configural account, but not the simple associative account, can explain monkeys' learning of visual-visual conditional discriminations and its sensitivity to prefrontal-temporal disconnection. In a visual-visual conditional discrimination, the monkey learns, for example, not only A-B-reward and C-D-reward but also A-D-nonreward and $\mathrm{C}-\mathrm{B}$-nonreward. This discrimination cannot be solved by acquiring two-term associations like A-B and B-reward. Rather, the monkey must learn that the unique visual configurations $\mathrm{A}-\mathrm{B}$ and
C-D are followed by reward. Uncinate fascicle section impairs visual-visual conditional learning (Gutnikov et al., 1997), and this supports the idea that both visual-visual conditional learning and visual serial compound learning depend on the same mechanism, namely, the acquisition of serial visual configural representations. The configural representation of simultaneous visual compounds, on the other hand, is not impaired by uncinate fascicle section (Gutnikov et al., 1997). Simultaneous visual configural learning depends critically on the perirhinal cortex, and the physiological and computational mechanisms for this specialization may be distinct from those required for elemental representations (Buckley et al., 1997; Buckley and Gaffan, 1998; Bussey and Saksida, 2002; Bussey et al., 2002a, 2005). Together, all these results suggest that monkeys process both serial and simultaneous visual compound events in a similar configural way, acquiring a configural representation of the whole event, but that the brain mechanisms of these configural representations differ in that only the serial configurations depend on prefrontaltemporal interaction.

Our hypothesis of an impairment in configural representation of temporally complex visual events can also explain other effects of prefrontal-temporal disconnection. For example, strategy alternation (Gaffan et al., 2002) and object discrimination learning set (Browning et al., 2007) both depend on temporally complex representations, because in those tasks normal performance depends on a representation of the present trial in configuration with immediately preceding (retrospective memory) or immediately upcoming (prospective memory) trials (Gaffan et al., 2002; Browning et al., 2007). In contrast, in concurrent object-reward association learning and in the unfilled delay task in the present experiment, only a temporally simple object-reward association is required. Similarly, in object-in-place scene learning, spatially distant elements of a scene, including background objects as well as choice objects, must be represented together even though they are seen in successive fixations (Browning et al., 2005).

Bilateral ablations of prefrontal cortex in the monkey produce impairments in a broad variety of cognitive tasks. Along with similar evidence from human neuroscience, this has led to the characterization of prefrontal cortex in terms of general cognitive abilities such as working memory (Goldman-Rakic, 1995), executive function (Miller and Cohen, 2001), or fluid intelligence and adaptive plasticity (Duncan, 2001). Instead, we suggest that the effects of bilateral prefrontal lesions are broad because all cognitive tasks require some form of temporally complex representation. The advantage of the temporal-frontal disconnection technique is that it can assess whether there is a need for prefrontal processing of one particular type of temporally complex representations, namely those that necessarily involve visual objects. When that type is not needed, as in the unfilled-delay task, no impairment is produced by the disconnection even though those tasks require fluid intelligence or working memory to the same extent that the two-item task does. 


\section{References}

Browning PG, Gaffan D (2008) Impairment in object-in-place scene learning after uncinate fascicle section in macaque monkeys. Behav Neurosci 122:477-482.

Browning PGF, Easton A, Buckley MJ, Gaffan D (2005) The role of prefrontal cortex in object-in-place learning in monkeys. Eur J Neurosci 22:3281-3291.

Browning PGF, Easton A, Gaffan D (2007) Frontal-temporal disconnection abolishes object discrimination learning set in macaque monkeys. Cereb Cortex 17:859-864.

Buckley MJ, Gaffan D (1998) Perirhinal cortex ablation impairs configural learning and paired-associate learning equally. Neuropsychologia 36:535-546.

Buckley MJ, Gaffan D, Murray EA (1997) Functional double dissociation between two inferior temporal cortical areas: perirhinal cortex versus middle temporal gyrus. J Neurophysiol 77:587-598.

Bussey TJ, Saksida LM (2002) The organization of visual object representations: a connectionist model of effects of lesions in perirhinal cortex. Eur J Neurosci 15:355-364.

Bussey TJ, Saksida LM, Murray EA (2002a) Perirhinal cortex resolves feature ambiguity in complex visual discriminations. Eur J Neurosci 15:365-374.

Bussey TJ, Wise SP, Murray EA (2002b) Interaction of ventral and orbital prefrontal cortex with inferotemporal cortex in conditional visuomotor learning. Behav Neurosci 116:703-715.

Bussey TJ, Saksida LM, Murray EA (2005) The perceptual-mnemonic/feature conjunction model of perirhinal cortex function. Q J Exp Psychol B 58:269-282.

Croxson PL, Johansen-Berg H, Behrens TEJ, Robson MD, Pinsk MA, Gross CG, Richter W, Richter MC, Kastner S, Rushworth MFS (2005) Quantitative investigation of connections of the prefrontal cortex in the human and macaque using probabilistic diffusion tractography. J Neurosci 25:8854-8866.

Duncan J (2001) An adaptive coding model of neural function in prefrontal cortex. Nat Rev Neurosci 2:820-829.

Eacott MJ, Gaffan D (1992) Inferotemporal-frontal disconnection: the uncinate fascicle and visual associative learning in monkeys. Eur J Neurosci 4:1320-1332.

Eacott MJ, Gaffan D, Murray EA (1994) Preserved recognition memory for small sets, and impaired stimulus identification for large sets, following rhinal cortex ablations in monkeys. Eur J Neurosci 6:1466-1478.

Easton A, Gaffan D (2001) Crossed unilateral lesions of the medial forebrain bundle and either inferior temporal or frontal cortex impair object- reward association learning in rhesus monkeys. Neuropsychologia 39:71-82.

Fuster JM, Bauer RH, Jervey JP (1985) Functional interactions between inferotemporal and prefrontal cortex in a cognitive task. Brain Res 330:299-307.

Gaffan D, Dickinson A (2008) Mediated generalization in discrimination learning by rhesus monkeys. Q J Exp Psychol 61:558-568.

Gaffan D, Eacott MJ (1995) Uncinate fascicle section leaves delayed matching-to-sample intact, with both large and small stimulus sets. Exp Brain Res 105:175-180.

Gaffan D, Hornak J (1997) Visual neglect in the monkey. Representation and disconnection. Brain 120:1647-1657.

Gaffan D, Murray EA (1990) Amygdalar interaction with the mediodorsal nucleus of the thalamus and the ventromedial prefrontal cortex in stimulus-reward associative learning in the monkey. J Neurosci 10:3479-3493.

Gaffan D, Easton A, Parker A (2002) Interaction of inferior temporal cortex with frontal cortex and basal forebrain: double dissociation in strategy implementation and associative learning. J Neurosci 22:7288-7296.

Goldman-Rakic PS (1995) Cellular basis of working memory. Neuron 14:477-485.

Gutnikov SA, Ma YY, Gaffan D (1997) Temporo-frontal disconnection impairs visual-visual paired association learning but not configural learning in Macaca monkeys. Eur J Neurosci 9:1524-1529.

Miller EK, Cohen JD (2001) An integrative theory of prefrontal cortex function. Annu Rev Neurosci 24:167-202.

MurrayEA, Gaffan D (2006) Prospective memory in the formation of learning sets by rhesus monkeys (Macaca mulatta). J Exp Psychol Anim Behav Process 32:87-90.

Parker A, Gaffan D (1998a) Memory after frontal/temporal disconnection in monkeys: conditional and non-conditional tasks, unilateral and bilateral frontal lesions. Neuropsychologia 36:259-271.

Parker A, Gaffan D (1998b) Interaction of frontal and perirhinal cortices in visual object recognition memory in monkeys. Eur J Neurosci 10:3044-3057.

Paxinos G, Huang X-F, Toga AW (2000) The rhesus monkey brain in stereotaxic coordinates. San Diego: Academic.

Petrides M, Pandya DN (2002) Association pathways of the prefrontal cortex and functional observations. In: Principles of frontal lobe function (Stuss DT, Knight RT, eds), pp 31-50. New York: Oxford UP.

Tanaka K (1996) Inferotemporal cortex and object vision. Annu Rev Neurosci 19:109-139.

Ungerleider LG, Gaffan D, Pelak VS (1989) Projections from inferior temporal cortex to prefrontal cortex via the uncinate fascicle in rhesus monkeys. Exp Brain Res 76:473-484. 